\title{
DESIGN OF A SMITH-PURCELL RADIATION BUNCH LENGTH DIAGNOSTIC
}

\author{
S.E. Korbly, W.J. Brown, M.A. Shapiro, and R.J. Temkin \\ Plasma Science and Fusion Center, MIT, Cambridge, MA 02139 USA
}

\begin{abstract}
We present the design of a Smith-Purcell radiation diagnostic to measure the micro bunch length of a $20 \mathrm{MeV}, 17$ $\mathrm{GHz}$ electron bunch train. The bunch length can be determined by measuring the frequency and angular distribution of the emitted radiation as proposed by Nguyen[1]. The beam is produced by a $17 \mathrm{GHz}, 50 \mathrm{MeV} / \mathrm{m}$ traveling wave (TW) accelerator built by Haimson Research Corporation (HRC). The high operating frequency of this accelerator allows for the production of ultra-short bunches of about 180 femtoseconds. We will present detailed calculations of the optimization of the experimental system and of the expected bunch length resolution.
\end{abstract}

\section{INTRODUCTION}

The HRC accelerator system consists of a chopperprebuncher injector, and a quasi-constant accelerating structure consisting of 94 cavities that operate in the $\frac{2 \pi}{3}$ mode[2]. The initial electron beam is produced by a 550 $\mathrm{kV}$ DC thermionic gun. A picture of the accelerator is shown in Figure 1. The HRC accelerator operating parameters are listed in Table 1. The accelerator can also be operated with an RF photocathode gun in place of the DC thermionic gun.

Table 1: HRC Accelerator Design Parameters

\begin{tabular}{|l|c|}
\hline Parameter & Value \\
\hline Beam Energy & $20-30 \mathrm{MeV}$ \\
Average Current & $0.25 \mathrm{~A}$ \\
Peak Current & $80 \mathrm{~A}$ \\
Electrons/bunch & $10^{8}$ \\
Emittance(Calc.) & $5 \pi \mathrm{mm}-\mathrm{mrad}$ \\
Bunch Length(Est.) & $55 \mu \mathrm{m}$ \\
\hline
\end{tabular}

Simulations [2] indicate that the phase spread at the exit of the accelerator should be about $1^{\circ}$ or a bunch length of $\sim 180$ fs. Several techniques have been employed to measure femtosecond bunch lengths including Optical Transition Radiation[3][4][5], Cherenkov Radiation[6], MicroWiggler[7] and streak cameras. We are proposing to use a Smith-Purcell bunch length diagnostic to measure the bunch length. Advantages of a Smith-Purcell diagnostic are that it is non-interfering, non-destructive, and affordable.

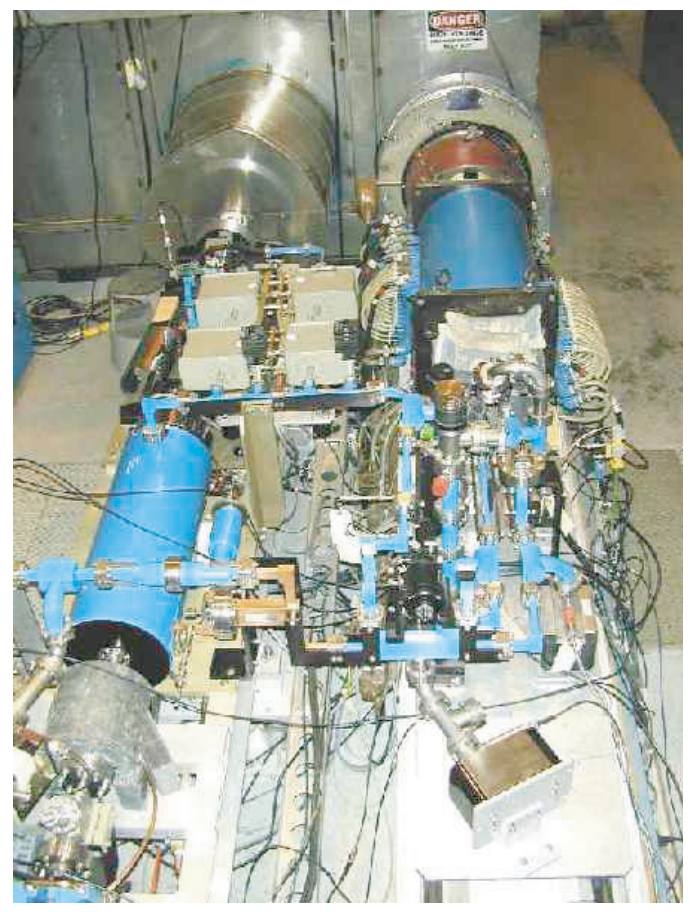

Figure 1: Picture of the linac (lower left), klystron (upper right), output arms and hybrid coupler (lower right) and electron guns (top).

\section{HRC ACCELERATOR}

The HRC accelerator is powered by a relativistic klystron[8]. The electron beam for the traveling-wave relativistic klystron (TWRK- Model X7100) powering the linac is space-charge limited and is produced by a thermionic Pierce gun built by Thomson CSF for MIT with a perveance of $0.27 \mu$ perv. The klystron was constructed as a high vacuum demountable assembly consisting of a compression input section, a drive and gain section, a bunching and TW output section, and an isolated beam collector. The rf power is coupled out of the tube by two WR62 rectangular waveguides. Measurements indicate that up to $95 \%$ of the $100 \mathrm{~A}$ beam can be transmitted through the klystron. The tube is designed to have a gain of between 60 and 70 $\mathrm{dB}$, and uses a $5 \mathrm{~W}$ pulsed TWTA to provide the drive signal. Klystron experiments into a matched load produced output powers up to $26 \mathrm{MW}$ with a saturated gain of 67 $\mathrm{dB}[8]$.

Both the accelerator and the relativisitic klystron are driven by the MIT high voltage modulator which produces 


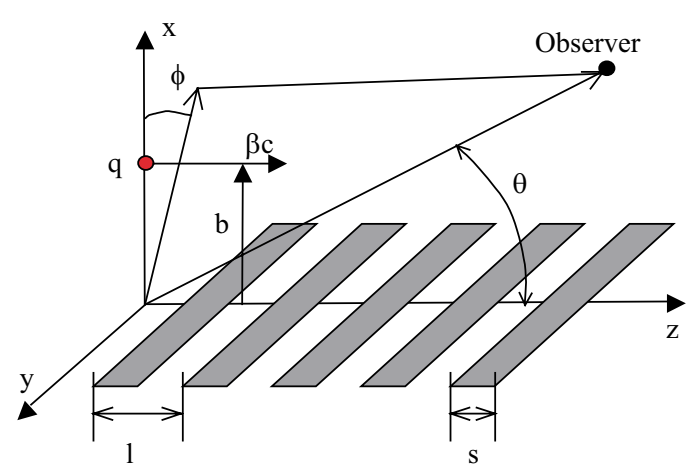

Figure 2: Schematic of an electron bunch, q, passing over a grating of period 1 , with an impact parameter $b$.

$1 \mu s$ pulses up to $700 \mathrm{kV}$ at a repetition rate of $4 \mathrm{~Hz}$. The linac consists of a chopper-prebuncher injector, and a quasi-constant accelerating structure consisting of 94cavities that operate in the $\frac{2 \pi}{3}$ mode. The electron bunches produced by the injector are designed to be accelerated by the linac to energies of 20-30 MeV. The linac can be used with either a DC or an RF gun. The first set of experiments were conducted with a $550 \mathrm{kV}$ DC thermionic gun [9]. Simulations indicate that the gun should produce a beam having a normalized RMS emittance between 2 and $3 \pi$ mm-mrad. Measurements of the emittance were completed using two collimators $(0.8$ and $3.4 \mathrm{~mm}$ radii) separated by $37 \mathrm{~cm}$. These measurements indicated that the gun was producing an average beam current of $0.94 \mathrm{~A}$ at $550 \mathrm{kV}$ with a normalized RMS emittance of approximately 1.8 $\pi$ mm-mrad, confirming that the DC gun does produce a high quality beam. This beam is well matched to the linac, which has an admittance of about $2 \pi$ mm-mrad.

Initial operation of the accelerator occurred without chopping or prebunching of the electron beam from the DC gun. RF powers up to $10 \mathrm{MW}$ were injected into the linac structure. Up to $100 \mathrm{~mA}$ of current was observed, confirming the high quality of the electron beam. In addition, an energy spectrometer verified beam energies up to 17.5 MeV, which is consistent with theoretical predictions. High power measurement of the RF filling time was also measured, and found to be about $60 \mathrm{~ns}$. This is consistent with the $0.032 \mathrm{c}$ design value for the harmonic group velocity of the linac structure. More recent results with the chopper and prebuncher are presented in [10].

\section{SMITH-PURCELL RADIATION DIAGNOSTIC}

An electron passing close to the surface of a metal diffraction grating as in Figure 2 emits Smith-Purcell radiation at a wavelength $\lambda_{n}$ given by

$$
\lambda_{n}=\frac{l}{n}\left(\frac{1}{\beta}-\cos \theta\right)
$$

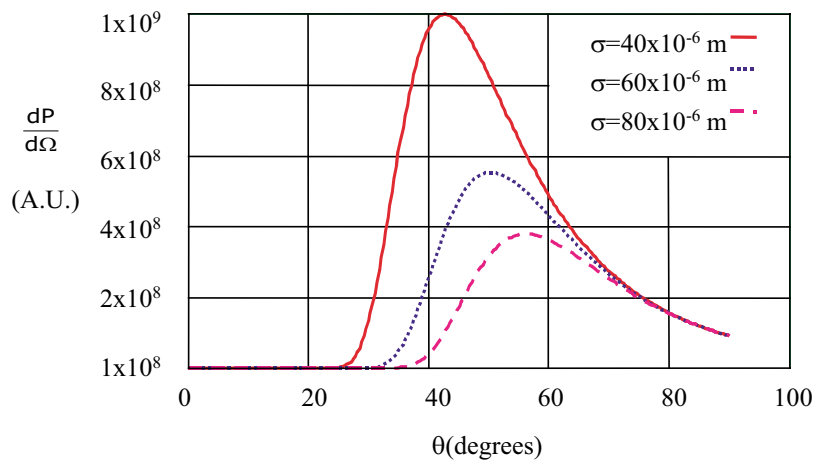

Figure 3: Plot of radiated power as a function of angle for three different bunch lengths $(40 \mu \mathrm{m}, 60 \mu \mathrm{m}$ and $80 \mu \mathrm{m})$.

where $l$ is the grating period, $\theta$ is the emission angle with respect to the propagation direction, $n$ is the diffraction order, and $\beta$ is the velocity of the electron bunch. The angular distribution of power radiated by the electrons is given by [11]

$$
\begin{aligned}
\frac{d P}{d \Omega} & =N_{g} \frac{e I n^{2} \beta^{3}}{2 l \varepsilon_{o}}\left(1+N_{e} e^{-k^{2} \sigma^{2} \cos ^{2} \theta}\right) \times \\
& {\left[\frac{\sin ^{2} \theta}{(1-\beta \cos \theta)^{3}}\right]\left|R_{n}^{2}\right| e^{-\left(\frac{4 \pi|n| b}{\gamma l(1-\beta \cos \theta)}\right)} }
\end{aligned}
$$

where $N_{g}$ is the number of grating strips, $I$ is the beam current, $N_{e}$ is the number of electrons in the bunch, $\varepsilon_{o}$ is the permittivity of free space, $b$ is the height of the bunch above the grating, $\gamma$ is the relativistic factor $\left(1-\beta^{2}\right)^{-\frac{1}{2}}$, $k$ is the wave vector, $\sigma$ is the bunch length, and $R_{n}^{2}$ is the grating efficiency factor.

For radiation at wavelengths shorter than the bunch length the radiation is incoherent. However, at wavelengths longer than the bunch length the radiation is coherent and the temporal coherence of the electron bunch enhances the intensity of the radiation. In general, the coherence term is $\left(1+N_{e} F\right)$, where $F$ is the form factor given by the Fourier transform of the bunch distribution. For Equation 2 a Gaussian distribution has been assumed giving a coherence term $\left(1+N_{e} e^{-k^{2} \sigma^{2} \cos ^{2} \theta}\right)$.

The estimated bunch length for the HRC accelerator is 180 femtoseconds or $54 \mu \mathrm{m}$. Equation 2 is plotted as a function of angle for three different bunch lengths (40 $\mu \mathrm{m}, 60 \mu \mathrm{m}$ and $80 \mu \mathrm{m})$ and for $b=100 \mu \mathrm{m}, \gamma=40$, $l=0.8 \mathrm{~mm}, \beta=0.999$ and $|n|=1$ in Figure 3. The shift in angular location of the radiation peak would be measurable.

Using the Smith-Purcell relationship, Equation 2 can be rewritten as a function of frequency giving 


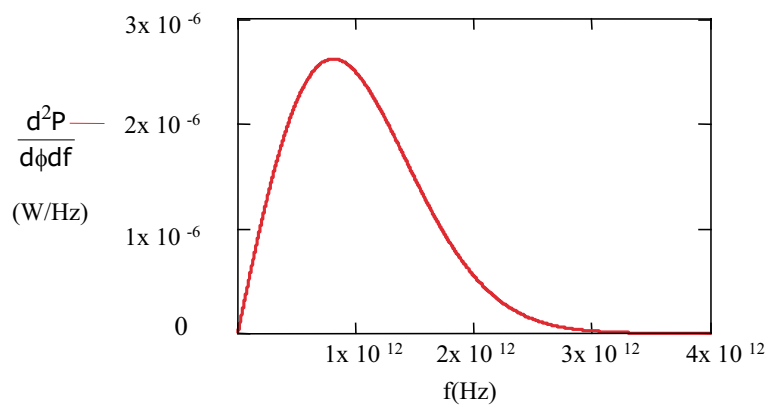

Figure 4: Plot of radiated power as a function of frequency.

$$
\begin{gathered}
\frac{d P}{d \phi d f}=\frac{e I L \beta^{3}}{2 \varepsilon_{o} c^{2}} f\left|R_{n}^{2}\right|\left(1+N_{e} e^{-\left(\frac{2 \pi f}{c}\right)^{2} \sigma^{2} \cos ^{2} \theta}\right) \times \\
\sin ^{2} \theta e^{-\left(\frac{4 \pi b f}{\gamma \beta c}\right)}
\end{gathered}
$$

Equation 3 is plotted in Figure 4 for $I=100 A, \gamma=40$, $\sigma=60 \mu \mathrm{m}, b=100 \mu \mathrm{m}, \theta=40^{\circ}$. The emitted radiation will be in the 0.5 to $1 \mathrm{THz}$ regime.

There are two conditions in order for the Smith-Purcell radiation to be of measurable intensity. The first requires the bunch to be close enough to the grating and the second requires the radiation wavelength to be shorter than the bunch length. In order to observe the effects of the bunch length rather than the beam height we require

$$
\frac{2 b}{\gamma}<\sigma \cos \theta
$$

For an impact parameter $b=100 \mu \mathrm{m}, \gamma=40, \frac{2 b}{\gamma}=$ $5 \mu \mathrm{m}$ and $\sigma=60 \mu \mathrm{m}$, Eq. 4 is satisfied. Preliminary TRACE3D [12] simulations indicate that the HRC beam can be focused to the required size using focusing magnets.

\section{CONCLUSIONS}

The $17 \mathrm{GHz}$ traveling wave disc loaded structure has demonstrated a beam energy of $17 \mathrm{MeV}$ at $100 \mathrm{~mA}$ current. To our knowledge this is the highest frequency stand alone accelerator in the world. We plan on implementing a Smith-Purcell radiation diagnostic to measure the bunch length of the HRC accelerator. The estimated bunch length of the accelerator is $\sim 180 \mathrm{fs}$ which should result in coherent radiation in the $\mathrm{THz}$ regime. In order to observe this radiation we will focus the beam over a diffraction grating using focusing magnets.

\section{ACKNOWLEDGEMENTS}

This research is supported by the U.S. Department of Energy.

\section{REFERENCES}

[1] D.C. Nguyen, "Electron Bunch Length Diagnostic With Coherent Smith-Purcell Radiation", PAC'97, Vancouver, May 1997

[2] J. Haimson and B. Mecklenburg, "HV injection and phase orbit characteristics for sub-picosecond bunch operation with a high gradient $17 \mathrm{GHz}$ linac", Proc. 6th Workshop on Advanced Accelerator Concepts, Lake Geneva, WI 1994

[3] P. Kung, et.al., "Generation and measurement of 50-fs(rms) electron pulses", Phys. Rev. Lett., Vol. 73, pp. 967-970 (1994)

[4] H. Lihn, et.al., "Measurement of subpicosecond electron pulses", Phys. Rev. E, Vol 53, pp 6413-6418 (1996)

[5] F. Amirmadhi, et.al., "Electron bunch length measurements at the Vanderbilt FEL" Nuclear Instruments and Methods in Physics Research A, Vol. 375, pp.95-6 (1996)

[6] J. Rosenzweig, et.al., "Initial measurements of the UCLA RF photoinjector", Nuclear Instruments and Methods in Physics Research A, Vol. 342, pp379-85 (1994)

[7] P. Catravas, et.al., "Measurement of Electron-Beam Bunch Length and Emittance Using Shot-Noise-Driven Fluctuations in Incoherent Radiation”, Phys. Rev. Lett., Vol 82, pp. 5261-5264 (1999)

[8] J. Haimson, B. Mecklenburg, B.G. Danly, 'Initial performance of a high gain, high efficiency $17 \mathrm{GHz}$ traveling wave relativistic klystron for high gradient accelerator research", Pulsed RF Sources for Linear Colliders. Montauk, NY, 1994

[9] J. Haimson, B. Mecklenburg, G. Stowell and E.L. Wright, "A fully Demountable $550 \mathrm{kV}$ Electron Gun for Low Emittance Beam Experiments with a $17 \mathrm{GHz}$ Linac", PAC'97, Vancouver, May 1997

[10] J. Haimson, B. Mecklenburg, G. Stowell, "Initial Performance of a $500 \mathrm{kV}$, Chopper Prebuncher Injection System for a $17 \mathrm{GHz}$ Linac", PAC'01, Chicago, June 2001

[11] K.J. Woods, J.E. Walsh, R.E. Stoner, H.G. Kirk and R.C. Fernow, "Forward Directed Smith-Purcell Radiation from Relativistic Electrons", Phys. Rev. Lett., Vol. 74, pp. 38083811 (1995)

[12] K.R. Crandall and D.P. Rusthoi, "Trace 3-D Documentation”, LANL (LA-UR-97-886) 1997 